\title{
Fuzzy Transportation Problem of Triangular Numbers with $\alpha$-Cut and Ranking Technique
}

\author{
Shugani Poonam ${ }^{1}$, Abbas S. H. ${ }^{2}$, Gupta V.K. ${ }^{3}$ \\ Institute for imsrastrure and human resource development, Vidisha (M.P.) \\ Saifia Science College Bhopal (M.P.) UIT, RGPV Bhopal (M.P.)
}

\begin{abstract}
The aim of Fuzzy transportation is to find the least transportation cost of some commodities through a capacitated network when the supply and demand of nodes and the capacity and cost of edges are represented as fuzzy numbers. Many previous papers $[1,2,5,6]$ have presented arithmetic operations, alpha level and simple ranking by operations. P. Pandian [4, 5] has presented some methods for fuzzy transportation problem. Finally all these research papers present the solutions of FTP by alpha level, simple ranking method and alpha level standard value. In this paper we are presenting a ranking technique with alpha optimal solution for solving fuzzy transportation problem, where fuzzy demand and supply all are in the form of triangular fuzzy numbers.
\end{abstract}

Keywords: - Fuzzy Transportation Problem, Triangular fuzzy numbers, $\alpha$ - optimal solution, Roubast Ranking Method.

\section{Introduction}

A fuzzy transportation problem is a transportation problem in which the transportation cost, supply and demand quantities are fuzzy quantities. The objective of the fuzzy transportation problem is to determine the shipping schedule that minimizes the total fuzzy transportation cost while satisfying fuzzy supply and demand limits.

In this paper we investigate more realistic problems, namely the transportation problem with fuzzy $\operatorname{costs} a_{i j}$. Since the objective is to minimize the total cost or to maximize the total profit, subject to some fuzzy constraints, the objective function is also considered as a fuzzy number. The method is to rank the fuzzy objective values of the objective function by some ranking method for numbers to find the best alternative. On the basis of this idea the Roubast Ranking method [7] with the help of $\alpha$ solution has been adopted a transform the fuzzy transportation problem. The idea is to transform a problem with fuzzy parameters in the form of Linear programming problem and solve it by the Vogel Approximation Method.

\section{Preliminaries}

Zadeh [9] in 1965 first introduced fuzzy set as a mathematical way of representing impreciseness or vagueness in everyday life.

Fuzzy set: A fuzzy set is characterized by a membership function mapping element of a domain, space or universe of discourse $X$ to the unit interval $[0,1]$ i.e. $A=\left\{\left(x, \mu_{A}(x) ; x \in X\right\}\right.$, Here $\mu_{A}: X \rightarrow[0,1]$ is a mapping called the degree of membership function of the fuzzy set $\mathrm{A}$ and $\mu_{\mathrm{A}}(\mathrm{x})$ is called the membership value of $\mathrm{x} \in \mathrm{X}$ in the fuzzy set $\mathrm{A}$. These membership grades are often represented by real numbers ranging from $[0,1]$.

Triangular fuzzy number: For a triangular fuzzy number A(x), it can be represented by A $(\mathrm{a}, \mathrm{b}, \mathrm{c} ; 1)$ with membership function $\mu(\mathrm{x})$ given by

$$
\left\{\begin{array}{cc}
(x-a) /(x-b) & a \leq x \leq b \\
1, & x=b \\
(c-x) /(c-d) & c \leq x \leq d \\
0, & \text { otherwise }
\end{array}\right.
$$

\section{Robust Ranking Technique:}

Roubast ranking technique which satisfy compensatio, linearity, and additively properties and provides results which are consist human intuition. If $\tilde{a}$ is a fuzzy number then the Roubast Ranking is defined by $\mathrm{R}(\tilde{\mathrm{a}})=\int_{0}^{1}(0.5)\left(a_{\alpha}^{L} a_{\alpha}^{U}\right) d \alpha$, where $\left(a_{\alpha}^{L} a_{\alpha}^{U}\right)$ is the $\alpha$ level cut of the fuzzy number a In this paper we use this method for ranking the objective values. The Roubast ranking index $R(\tilde{a})$ gives the representative value of fuzzy number ã. 


\section{Numerical Example:-}

A company has four sources $S_{1}, S_{2}, S_{3}$ and $S_{4}$ and four destinations $D_{1}, D_{2}, D_{3}$ and $D_{4}$; the fuzzy transportation cost for unit quantity of the product from $\mathrm{i}^{\text {th }}$

source to $j^{\text {th }}$ destination is $\mathrm{C}_{\mathrm{ij}}$ where $\left[c_{i j}\right]_{4 \times 4}=\left(\begin{array}{c}(5,10,15)(5,10,20)(5,15,20)(5,10,15) \\ (5,10,20)(5,15,20)(5,10,15)(10,15,20) \\ (5,10,20)(10,15,20)(10,15,20)(5,10,15) \\ (10,15,25)(5,10,15)(10,20,30)(10,15,25)\end{array}\right)$

And fuzzy availability of the product at source are $((10,15,20),(5,10,15),(20,30,40),(15,20,25))$ and the fuzzy demand of the product at destinations are $((25,30,35),(10,15,20),(5,15,20)(10,15,25))$ respectively.

The fuzzy transportation problems are

Table-1

\begin{tabular}{|l|l|l|l|l|}
\hline$(5,10,15)$ & $(5,10,20)$ & $(5,15,20)$ & $(5,10,15)$ & $(10,15,20)$ \\
\hline$(5,10,20)$ & $(5,15,20)$ & $(5,15,20)$ & $(10,15,20)$ & $(5,10,15)$ \\
\hline$(5,10,20)$ & $(10,15,20)$ & $(10,15,20)$ & $(5,10,15)$ & $(20,30,40)$ \\
\hline$(10,15,25)$ & $(5,10,15)$ & $(10,20,30)$ & $(10,15,25)$ & $(15,20,25)$ \\
\hline$(25,30,35)$ & $(10,15,20)$ & $(5,15,20)$ & $(10,15,25)$ & \\
\hline
\end{tabular}

Solution: In Conformation to model the fuzzy transportation problem can be formulated in the following mathematical programming form

Min $Z=R(5,10,15) x_{11}+R(5,10,20) x_{12}+R(5,15,20) x_{13}+R(5,10,15) x_{14}+R(5,10,20) x_{21}+R(5,15,20) x_{22}+R(5,10,15) x_{23}+$ $\mathrm{R}(5,10,20) \mathrm{x}_{24}+\mathrm{R}(5,10,20) \mathrm{x}_{31}+\mathrm{R}(10,15,20) \mathrm{x}_{32}+\mathrm{R}(10,15,20) \mathrm{x}_{33}+\mathrm{R}(5,10,15) \mathrm{x}_{34}+\mathrm{R}(10,15,25) \mathrm{x}_{41}+\mathrm{R}(5,10,15) \mathrm{x}_{42}+$ $\mathrm{R}(10,20,30) \mathrm{x}_{43}+\mathrm{R}(10,15,25) \mathrm{x}_{44}$

Where

$$
R(\bar{a})=\int_{0}^{1}(0.5)\left(a_{\alpha}^{L}, a_{\alpha}^{U}\right) d \alpha
$$

Similarly

$$
\begin{array}{r}
R(5,10,20)=11.25, R(5,15,20)=13.75, R(5,10,15)=10, R(5,10,20)=11.25 \\
R(5,15,20)=13.75, R(5,10,15)=10, R(10,15,20)=15, R(5,10,20)=11.25 \\
R(10,15,20)=15, R(10,15,20)=15, R(5,10,15)=10, R(10,15,25)=16.25 \\
R(5,10,15)=10, R(10,20,30)=20, R(10,15,25)=16.25
\end{array}
$$

\begin{tabular}{|c|c|c|c|c|c|}
\hline & FD1 & FD2 & FD3 & FD4 & $\begin{array}{l}\text { Fuzzy } \\
\text { Capacity }\end{array}$ \\
\hline FO1 & $\begin{array}{ll}\mathbf{1 1 . 2 5} \\
10\end{array}$ & 11.25 & $13.75^{\mathbf{3 . 7 5}}$ & 10 & 15 \\
\hline FO2 & 11.25 & 13.75 & $\begin{array}{ll}10 & \mathbf{1 0} \\
\end{array}$ & 15 & 10 \\
\hline FO3 & $11.25^{\mathbf{1 3 . 7 5}}$ & 15 & 15 & $10 \begin{array}{ll}16.25 \\
10\end{array}$ & 30 \\
\hline FO4 & $16.25^{\mathbf{5}}$ & $\begin{array}{ll} & \mathbf{1 5} \\
10 & \end{array}$ & 20 & 16.25 & 20 \\
\hline Fuzzy demand & 30 & 15 & 13.75 & 16.25 & \\
\hline
\end{tabular}

Rank of all supply $R(10,15,20)=15, R(5,10,15)=10, R(20,30,40)=30, R(15,20,25)=20$

Rank of all Demands $R(25,30,35)=30, R(10,15,20)=15, R(5,15,20)=13.75, R(10,15,25)=16.25$

\section{Table-2}


Table-3

\begin{tabular}{|c|c|c|c|c|c|}
\hline & FD1 & FD2 & FD3 & FD4 & $\begin{array}{l}\text { Fuzzy } \\
\text { Capacity }\end{array}$ \\
\hline FO1 & $\begin{array}{c}\mathbf{( 1 0 , 1 5 , 2 0 )} \\
(5,10,15)\end{array}$ & $(5,10,20)$ & $(5,15,20)$ & $(5,10,15)$ & $(10,15,20)$ \\
\hline FO2 & $(5,10,20)$ & $(5,15,20)$ & $\begin{array}{c}(\mathbf{5 , 1 0 , 1 5 )} \\
(5,10,15)\end{array}$ & $(10,15,20)$ & $(5,10,15)$ \\
\hline FO3 & $\begin{array}{l}\mathbf{( - 1 0 , 1 0 , 3 0 )} \\
(5,10,20)\end{array}$ & $(10,15,20)$ & $(10,15,20)$ & $\begin{array}{c}(\mathbf{1 0 , 1 5 , 2 5 )} \\
(5,10,15)\end{array}$ & $(20,30,40)$ \\
\hline FO4 & $\begin{array}{r}\mathbf{( - 5 , 5 , 1 5 )} \\
(10,15,25) \\
\end{array}$ & $\begin{array}{l}(\mathbf{1 0 , 1 5 , 2 0}) \\
(5,10,15)\end{array}$ & $(10,20,30)$ & $(10,15,25)$ & $(15,20,25)$ \\
\hline Fuzzy demand & $(25,30,35)$ & $(10,15,20)$ & $(5,15,20)$ & $(10,15,25)$ & \\
\hline
\end{tabular}

In the above examples it has been shown that the total optimal cost obtained by our methods remains as that obtained by defuzzifying the total fuzzy optimal cost by applying the Robust's Ranking method.

RESULT: For the fuzzy transportation problem with fuzzy objective function min $\mathrm{z}=812.5$

\section{CONCLUSION}

In this paper, the transportation costs are considered as imprecise numbers described by fuzzy numbers which are more realistic and general in nature. Moreover, the fuzzy transportation problem of triangular numbers has been transformed into crisp transportation problem using Robust's ranking indices ${ }^{5}$. Numerical examples show that by this method we can have the optimal solution as well as the crisp and fuzzy optimal total cost. By using Robust's ${ }^{5}$ ranking method we have shown that the total cost obtained is optimal. Moreover, one can conclude that the solution of fuzzy problems can be obtained by Robust's ranking method effectively. This technique can also be used in solving other types of problems like, project schedules, assignment problems and network flow problems.

\section{Refrences}

[1] Lin, Feng_Tse and Tsai, Tzong_Ru:“A two stage genetic algorithm for solving the transportation problem with fuzzy demands and fuzzy supplies", Int. J. of innovative computing information and control. Vol.5. (2009)

[2] Liu, Shiang-Tai and Chiang, Kao: "Solving fuzzy transportation problems based on extension principle", European Journal of Operational Research, 153, pp 661-674 (2004).

[3] Pandian, P. and Natrajan, G.: "An optimal More for less solution to fuzzy transportation problem with mixed constraints" Applied mathematical sciences, vol.4, no.29, 1405-1415 (2010).

[4] Pandian, P. and Natrajan, G.: "A new algorithm for finding a fuzzy optimal solution for fuzzy transportation problem', applied mathematical sciences, vol.4, No.2, 79-90 (2010).

[5] R.R.Yager,: "A procedure for ordering fuzzy subsets of the unit interval”, Information Sciences, 24, 143-161 (1981).

[6] Ritha, W. and Vinotha, J. Merline: "Multi-objective two stage fuzzy transportation problem", journal of physical science, vol. 13, pp 107-120 (2009).

[7] R. Nagarajan and A.Solairaju: "Computing Improved Fuzzy Optimal Hungarian Assignment Problems with Fuzzy Costs under Robust Ranking Techniques" international journal of computer application, vol 6, no. 4 (2010)

[8] Sonia, and Malhotra, Rita: "A polynomial algorithm for a two stage time minimising transportation problem", OPSEARCH, 39, n0.5 and 6, pp 251-266 (2003).

[9] Zadeh, L. A: "Fuzzy sets, Information and Control", 8, pp 338-353 (1965). 